\title{
La Détention Des Mineurs Etrangers Non Accompagnés Dans Le Contexte De L’immigration
}

\author{
Mouna Haddad
}

Université Laval

\begin{abstract}
Résumé
Dans plusieurs pays à travers le monde certaines autorités gouvernementales arrêtent des mineurs étrangers non accompagnés et les détiennent dans des centres fermés. En général leur détention n'est pas relative à une infraction pénale, mais au manque de documents règlementaires requis qui rendraient possible leur entrée légale dans le pays. Il est particulièrement important que les États membres à la Convention relative aux droits de l'enfant reconnaissent aux mineurs étrangers non accompagnés le droit d'être traités avec humanité et avec respect de leur qualité d'enfant, tout en tenant compte de leurs besoins spécifiques. Dans le présent article, nous exposons d'abord les faits d'une affaire célèbre survenue en Belgique concernant la détention d'une mineure étrangère non accompagnée, âgée de cinq ans à l'époque. On constate avec cette affaire que la problématique de la détention des mineurs étrangers non accompagnés est pertinente aussi dans d'autres pays que le Canada. Nous abordons ensuite la norme internationale sur la détention des enfants garantie dans la Convention relative aux droits de l'enfant, le but est d'examiner la conformité à cette norme des dispositions concernant la détention des mineurs contenues dans la Loi sur l'immigration et la protection des réfugiés du Canada.
\end{abstract}

Mots clés: Convention relative aux droits de l'enfant, mineur étranger non-accompagné, détention, immigration, protection, Canada. 


\begin{abstract}
In many countries in the world certain governmental authorities arrest unaccompanied foreign minors and detain them in closed centres. Generally it is not because they have committed a criminal offence, but rather that they lack valid administrative papers allowing them to enter the country legally. It is particularly important that State Parties to the Convention on the Rights of the Child recognize that unaccompanied foreign minors have the right to be treated with humanity and as children with specific needs. In this article, we will first recount a famous Belgian case concerning the detention of an unaccompanied foreign minor who was five-years old at the time. This case will highlight that the problem of the detention of unaccompanied foreign minors is relevant to other countries beside Canada. We then explore the international standards with regard to children's detention established by the Convention on the Rights of the Child. The objective is to examine whether the Canadian Immigration and Refugee Protection Act's position on detention complies with this norm.
\end{abstract}

Keywords: Convention on the rights of the child, unaccompanied foreign minor, detention, immigration, protection, Canada.

\title{
Introduction
}

Le 18 août 2002, une décision de refus d'accès au territoire avec refoulement fut prise par les autorités belges à l'encontre d'une enfant congolaise de 5 ans, le motif étant qu'elle n'était pas en possession des documents requis par la loi belge sur l'accès au territoire des étrangers ${ }^{\mathrm{ii}}$. Le même jour une décision de son maintien dans un lieu de rétention situé à la frontière fut prise. L'enfant a été placée en détention dans le centre fermé 127bis. Elle a été expulsée sans accompagnement par les autorités belges vers son pays d'origine alors qu'elle cherchait tout simplement à joindre sa mère qui l'attendait au Canada. Cette affaire dite Tabitha, tirée du nom de l'enfant, a donné lieu à une condamnation de la Belgique par la Cour européenne des droits de l'homme ${ }^{\mathrm{iii}}$. Elle a contribué à accélérer le processus d'adoption de la Loi-programme du 24 décembre 2002 sur la tutelle des mineurs étrangers non accompagnés $^{\text {iv }}$. Cette loi a pour but de mettre en place un système de protection et de représentation spécifique pour ces mineurs et institue à cet effet un service de Tutelles 
indépendant. La Belgique n'est pas le seul pays concerné par l'arrivée de mineurs étrangers non accompagnés sur son sol. D’autres pays, dont le Canada, auquel nous consacrerons cet article, sont également confrontés à ce phénomène qui mérite une réponse juridique adéquate en termes de protection de l'enfance.

Par mineur étranger non accompagné, on entend un enfant au sens de l'article premier de la Convention relative aux droits de l'enfant $(C R D E)^{v}$ qui a été séparé de ses deux parents ainsi que d'autres membres proches de sa famille, et qui n'est pas pris en charge par un adulte investi de cette responsabilité par la loi ou la coutume ${ }^{\mathrm{vi}}$. Ces mineurs arrivent dans le pays d'accueil sans documents administratifs requis et leur situation nécessite une régularisation. À leur arrivée, certains pays leur permettent de faire une demande d'asile ou toute autre demande permettant d'obtenir une autorisation à entrer et séjourner sur leur territoire.

Différentes raisons amènent le mineur non accompagné à se déplacer seul en dehors de son pays d'origine ou du pays de sa résidence habituelle : la persécution, la traite, la vente de l'enfant par ses parents, un conflit armé, une guerre civile ou tout simplement la recherche d'une meilleure vie loin de la pauvreté et de la famine. Le mineur étranger non accompagné se retrouve bien souvent livré à lui-même, sans repères culturels et familiaux solides ce qui fait de lui un être plus vulnérable. Il est donc exposé au risque d'exploitation, d'abus sexuel ou de détention et mérite qu'on lui prête une attention toute particulière. Néanmoins, sa caractéristique d'être étranger est un obstacle non négligeable à sa protection par l'État d'accueil mais cela ne justifie pas un éloignement de la norme internationale et quasi universelle relative à la protection des enfants.

Le présent article a pour objectif l'examen de la norme internationale sur la détention des enfants garantie par la Convention relative aux droits de l'enfant $(\boldsymbol{I})$. Cette analyse permettra de vérifier la conformité à cette norme des dispositions de la Loi sur l'immigration et la protection des réfugiés ${ }^{\mathrm{vii}} \mathrm{du}$ Canada (II).

\section{I: La norme internationale concernant la détention des enfants}

Dans la Convention relative aux droits de l'enfant, la détention est règlementée à l'article 37 (I.1).

\section{I.1. L'article 37 de la Convention relative aux droits de l'enfant}

Toute forme de privation de liberté est une atteinte au droit de tout être humain à la liberté, que ce soit un adulte ou un enfant. Ce droit fut reconnu d'abord par l'article 9(1) du 
Pacte international relatif aux droits civils et politiques. On peut remarquer que ce pacte permet une certaine restriction du droit à la liberté, mais seulement si la détention n'est pas arbitraire.

En droit international des droits de l'enfant c'est notamment la Convention relative aux droits de l'enfant ${ }^{\text {viii }}$ qui contient une disposition équivalente qui est l'article 37 (b) selon lequel:

37) « Les États parties veillent à ce que :

a) $\ldots$

b) Nul enfant ne soit privé de liberté de façon illégale ou arbitraire.

L'arrestation, la détention ou l'emprisonnement d'un enfant doit être en conformité avec la loi, n'être qu'une mesure de dernier ressort, et être d'une durée aussi brève que possible;

c)...

d)...»

L'article 37 utilise le terme privé de liberté sans préciser la raison de la privation de la liberté de l'enfant. En conséquence, l'application de l'article 37 n'est pas limitée aux enfants détenus en raison de la commission d'une infraction pénale, mais s'étend aussi à la détention administrative des mineurs étrangers non accompagnés en vertu des lois nationales sur l'immigration.

Le Comité des droits de l'enfant des Nations unies, responsable de la bonne mise en œuvre de la CRDE et de son interprétation, s'appuie sur l'article 37 pour définir les conditions de détention des mineurs étrangers non accompagnés. Le Comité précise qu' «en application de l'article 37 de la Convention et du principe d'intérêt supérieur de l'enfant, les enfants non accompagnés ou séparés ne devraient pas, en règle générale, être placés en détention» ${ }^{\mathrm{ix}}$. En conséquence, même si la détention du mineur étranger non accompagné n'est pas interdite en soi, le principe mentionné est que la détention ne doit être qu'une mesure de dernier ressort. Cela implique que la détention doit être une mesure nécessaire. L'article 37(b) ajoute que le délai de la détention doit être aussi bref que possible. La fixation d'un délai maximale de détention des mineurs devrait, à notre avis, être expressément garantie dans la loi.

Le Comité des droits de l'enfant ajoute que «la détention ne saurait être justifiée par le seul fait que l'enfant est séparé ou non accompagné, ni par son seul statut au regard de la législation relative à l'immigration ou à la résidence ou l'absence d'un tel statut ${ }^{\mathrm{x}}$. Même si la loi nationale sur l'immigration prévoit la possibilité de détenir le mineur étranger pour le 
motif de séjour irrégulier, une telle loi est contraire aux recommandations du Comité des droits de l'enfant. Le Comité exige quelque chose de plus que ce motif de détention lié au manque de documents règlementaires valides autorisant l'entrée dans le pays. Cela peut être, par exemple, le fait que le mineur étranger présente un danger concret et réel pour le public, ce qui n'est pas le cas de la majorité des mineurs étrangers non accompagnés.

Par ailleurs, l'article 37(c) de la CRDE impose à la charge des États membres une obligation positive de garantir des conditions minimales de détention. Ces conditions permettent d'assurer que les enfants détenus soient traités avec humanité et avec le respect de leur dignité en répondant à leurs besoins spécifiques de leur qualité d'enfant:

«37 c) Tout enfant privé de liberté soit traité avec humanité et avec le respect dû à la dignité de la personne humaine, et d'une manière tenant compte des besoins des personnes de son âge».

Cette norme garantit également que « tout enfant privé de liberté sera séparé des adultes, à moins que l'on estime préférable de ne pas le faire dans l'intérêt supérieur de l'enfant, ...» ${ }^{\mathrm{xi}}$.

\section{Le cadre légal canadien de la détention des mineurs étrangers non accompagnés dans le contexte de l'immigration}

À l'heure actuelle, l'État canadien ne dispose pas de statistiques centrales et unifiées sur les mineurs étrangers non accompagnés qui arrivent à différents points d'entrée du Canada (II.1). Si les mineurs sont repérés par la police des frontières, ils peuvent être détenus en vertu de la loi sur l'immigration et la protection des réfugiés, une détention qui devrait néanmoins se faire conformément à la norme internationale (II.2).

\section{II.1. L'absence de données statistiques centrales sur la détention des mineurs étrangers non accompagnés}

Au Canada, nous n'avons pas de données officielles et centralisées sur le nombre de mineurs étrangers non accompagnés qui sont détenus dans les centres de rétention en vertu de la loi sur l'immigration et la protection des réfugiés ${ }^{x i i}$.Claudette Deschênes, vice présidente de l'agence des services frontaliers du Canada avait témoigné devant le Comité sénatorial 
permanent des droits de la personne et avait mentionné «qu'en 2005-2006, il y avait 715 enfants migrants qui avaient été détenus au Canada, dont 70 p. 100, pendant moins de six jours. Parmi ces enfants, 620 étaient accompagnés et 95 enfants non accompagnés » ${ }^{\text {iii }}$. Caroline Montpetit estime qu'il y a au moins 430 enfants demandeurs d'asile par année qui sont détenus dans des prisons canadiennes en vertu d'un pouvoir discrétionnaire des agents des services frontaliers du Canadaxiv. De même, le Conseil canadien pour les réfugiés prétend que les enfants étrangers en séjour irrégulier sont régulièrement détenus au Canada, parfois pour une période de plusieurs semaines, et cela même quand ils ne représentent aucun risque pour la sécurité ni aucun danger pour le public ${ }^{\mathrm{xv}}$. Mais nous ne pouvons pas vérifier les données que le Conseil canadien pour les réfugiés fournit. Il faut donc les prendre avec précautions. À ce titre, l'absence d'une base de données centralisée et officielle sur les mineurs étrangers non accompagnés au Canada est regrettable. Une telle base de données nous permettrait de les confronter aux données fournies par les organisations non gouvernementales et de vérifier leur conformité, car ces organisations peuvent avoir des intérêts personnels et financiers à diffuser de telles données, ce qui nuit à leur crédibilité. En l'absence de la base de données gouvernementale, il nous est donc compliqué d'avoir accès à la réalité de la situation des mineurs non accompagnés au Canada. La seule conclusion que nous pouvons tirer de cet état de choses est que le Canada détient des mineurs étrangers non accompagnés en centre fermé. En effet, nous ne pouvons pas avoir une meilleure estimation de la situation, car nous n'avons pas d'information officielle centralisée concernant le nombre exact de mineurs étrangers détenus, la durée, le motif et les conditions de détention.

Finalement, nous ne savons pas si leur droit fondamental à l'éducation et aux soins de santé est respecté pendant leur détention.

Il est souhaitable donc corriger l'absence de statistiques centrale, d'autant plus que le Comité des droits de l'enfant des Nations unies avait lui-même constaté que des mineurs étrangers non accompagnés continuent à être détenus au Canada en vertu de la loi canadienne sur l'immigration.

En 1995, le Comité des droits de l'enfant avait recommandé au gouvernement fédéral d'examiner la situation des enfants qui se sont vus refuser le statut de réfugié et qui sont en attente de leur expulsion $^{\text {xvi }}$. En 1999, la situation commence à changer au Canada à cause de l'arrivée en Ontario d'un groupe de mineurs étrangers d'origine chinoise âgés de 11 à 17 ans non accompagnés qui étaient exploités par des trafiquants. Ces enfants ont été détenus pour des périodes allant parfois jusqu'à sept mois. Les motifs de ces détentions prolongées étaient qu'ils constituaient un danger pour le public ou qu'ils risquaient de s'enfuir. Leurs conditions 
de détention ne leur accordaient aucune protection contre l'environnement risqué dans lequel ils étaient détenus. En effet ils étaient placés avec des adultes criminels en centres fermés et rien n'a été mis en place pour les défendre contre cet environnement inadéquat pour un enfant. Ils n'ont eu aucun service qui aurait permis de combler leurs besoins spéciaux d'ordre psychologiques, physiques, médicaux, éducatifs et de loisirs ${ }^{\text {xvii }}$. Depuis cet événement, le Canada a modifié la Loi sur l'immigration et la protection des réfugiés (LIPR) de façon à y inclure le principe de l'intérêt supérieur de l'enfant. Néanmoins, ce principe clé garanti par l'article 3 de la CRDE ne figure pas dans toutes les dispositions de la LIPR concernant les enfants.

\section{II.2 La loi sur l'immigration et la protection des réfugiés}

La Cour fédérale avait clarifié que «dans la société canadienne la détention constitue une restriction extraordinaire» ${ }^{\text {xiii }}$. À ce jour, la détention des étrangers dans le contexte de l'immigration est réglementée à la section 6 de la loi sur l'immigration et la protection des réfugiés $^{\text {xix }}$ (LIPR) à partir de l'article 54 qui donne compétence à la section de l'immigration de contrôler la détention des étrangers.

La détention des mineurs étrangers est réglementée à l'article 60 de la LIPR selon lequel:

«60. Pour l'application de la présente section, et compte tenu des autres motifs et critères applicables, y compris l'intérêt supérieur de l'enfant, est affirmé le principe que la détention des mineurs doit n'être qu'une mesure de dernier recours ».

Cette disposition, à priori conforme à l'article 37 de la CRDE, incorpore le principe de l'intérêt supérieur de l'enfant dans les décisions concernant sa détention et affirme que la détention d'un mineur ne doit être qu'une mesure de dernier recours. L'article 60 n'exclut pas la détention du mineur étranger, en raison des motifs de détention applicables aux étrangers adultes, lesquels sont précisés à l'article 55 de la LIPR. Il y avait au départ quatre motifs facultatifs de détention auxquels fut ajouté un cinquième motif de détention qui oblige l'agent à détenir l'étranger. 


\section{Les quatre motifs facultatifs de détention}

L'article 55 de la LIPR définit quatre motifs facultatifs permettant la détention d'un étranger ${ }^{\mathrm{xx}}$ : le danger pour la sécurité publique, le risque de fuite de l'étranger, la demanded'enquête du ministre de la Citoyenneté et de l'Immigration lorsqu'il soupçonne que l'étranger est interdit du territoire pour des raisons de sécurité ou d'atteintes aux droits humains ou internationaux, et le motif de l'identité non établie de l'étranger. Pour tous ces motifs, la jurisprudence a établi une liste non exhaustive de facteurs appelés facteurs $\operatorname{Sahin}^{\mathrm{xxi}}$ dont le décideur doit tenir compte au moment où il rend une décision de détention ou de mise en liberté dans le contexte de l'immigration, que ce soit pour un majeur ou un mineur. Ces facteurs ont été codifiés ensuite à l'article 248 du Règlement sur l'immigration et la protection des réfugiés $(R I P R)^{x x i i}$.

\section{L'ajout d'un cinquième motif de détention obligatoire par le projet de loi C-4}

Avec l'adoption relativement récente du projet de loi C-4, qui est problématique pour les mineurs étrangers non accompagnés en séjour irrégulier, un cinquième motif de détention fut ajouté et inclus à l'article 55(3.1) de la LIPR ${ }^{\text {xxiii }}$. Il s'agit du «défaut d'établir l'identité de l'étranger désigné qui était âgé de seize ans ou plus à la date de l'arrivée visée par la désignation en cause » ${ }^{\mathrm{xiv}}$.

Le projet de loi C-4 émanant du ministre de la Sécurité publique ${ }^{\mathrm{xxv}}$ avait créé une nouvelle catégorie dite d'étrangers désignés insérée dans l'article 20.1 (1) de la LIPR. Cette nouvelle disposition permet au ministre de la Sécurité publique, lorsque l'intérêt public l'exige, de désigner l'arrivée d'un groupe de personnes étrangères au Canada comme une « arrivée irrégulière ». Les membres de ce groupe deviennent alors des «étrangers désignés » en raison de leur arrivée irrégulière. En conséquence, ils doivent subir toutes les restrictions apportées par le projet de loi adopté. Leur détention est désormais rendue obligatoire comme le prévoit l'article 55 (3.1) de la LIPR :

«Art. 55 Arrestation et détention obligatoires - étranger désigné

(3.1) lorsqu'une désignation est faite en vertu du paragraphe 20.1(1), l'agent, selon le cas :

a) détient, à son entrée au Canada, l'étranger qui est un étranger désigné en conséquence de la désignation et qui est âgé de seize ans ou plus à la date de l'arrivée visée par la désignation; 
b) arrête et détient, sans mandat, l'étranger qui, après son entrée, devient un étranger désigné en conséquence de la désignation et qui était âgé de seize ans ou plus à la date de l'arrivée visée par la désignation ou lance un mandat pour son arrestation et sa détention ».

Un mineur étranger non accompagné qui arrive de manière irrégulière avec des passeurs contre qui le projet de loi est destiné en premier, serait-il aussi considéré comme appartenant à ce groupe d'étrangers désignés et détenus obligatoirement par les agents des frontières? Le projet de loi adopté n'introduit aucune nuance ou exception en ce qui concerne les enfants en séjour irrégulier. Au contraire, il inclut expressément les mineurs âgés de 16 ans et plus dans l'article 55(3.1). À l'instar des adultes, la détention des mineurs étrangers non accompagnés âgés de seize ans ou plus devient automatique et obligatoire. Nous sommes d'avis que ce caractère obligatoire de la détention des mineurs contredit l'article 60 de la même LIPR selon lequel la détention d'un mineur ne doit être qu'une mesure de dernier ressort. Comment envisager la détention d'un mineur comme une mesure de dernier ressort lorsqu'elle est obligatoire ?! Le caractère obligatoire de la détention est également contraire à l'article 37 de la CRDE et aux lignes directrices du Comité des droits de l'enfant et même aux lignes directrices du Haut Commissariat aux réfugiés et aux apatrides sur la détention des mineurs demandeurs d'asile ${ }^{\mathrm{xxvi}}$.

Le projet de loi adopté instaure du même coup des conditions de mise en liberté plus difficiles pour les «étrangers désignés » et d'autres restrictions applicables à cette catégorie d'étrangers comme l'impossibilité de demander la résidence permanente pendant au moins 5 ans après leur désignation ${ }^{\text {xxvii }}$. Par ailleurs, le projet de loi adopté n'accorde pas aux «étrangers désignés » le droit d'appel à la Section d'appel des réfugiés. Au contraire, il rend plus difficile le contrôle de la détention des étrangers désignés et les conditions de leur libération. L'actuel article 57.1, $§ 1$ de la LIPR rend impossible un premier contrôle des motifs de détention par la section d'appel avant l'expiration d'un délai de quatorze jours, ce qui est énorme pour un mineur. Après l'expiration de quatorze jours, l'article prévoit un contrôle dans les meilleurs délais. Cette disposition consacrée au groupe d'étrangers désignés est une exception à l'article 57(1) de la LIPR qui permet un premier contrôle des motifs de détention dans les 48 heures suivant la détention. Elle est également une exception à l'article 57(2) de la LIPR qui permet des contrôles supplémentaires au moins une fois tous les sept jours suivants le premier contrôle, ensuite tous les 30 jours. 
S'il semble compréhensible que l'objet principal du projet de loi C-4 soit de prévenir l'organisation de l'entrée illégale et l'arrivée massive de migrants irréguliers au Canada ${ }^{\mathrm{xxviii}}$, sa lacune consiste à ne faire aucune distinction, exception ou nuance concernant les mineurs étrangers non accompagnés âgés de seize à dix-sept ans par rapport aux adultes étrangers désignés.

\section{La durée de la détention}

La LIPR ne prévoit aucune durée maximale pour la détention des étrangers mineurs ou majeurs. Tout ce qu'elle prévoit c'est la possibilité d'avoir un contrôle judiciaire de la durée de détention, ce qui n'est pas comparable à une disposition législative fixant une limite maximale de la durée de détention. Le fait d'inclure une telle disposition dans la LIPR liera les tribunaux et limitera leur pouvoir plus ou moins discrétionnaire à fixer par eux-mêmes la durée maximale de la détention de l'étranger. Cela est encore plus pertinent lorsque l'étranger est un mineur. C'est pourquoi nous invitons le législateur fédéral à inclure une telle disposition qui fait défaut dans l'actuelle loi.

Conformément à l'article 56(2) de la LIPR, un étranger désigné peut être libéré uniquement dans trois cas: si sa demande d'asile ou de protection est admise en dernier ressort, ou en cas de la prise d'effet d'une mesure de liberté prononcée en vertu de l'article 58, ou en cas de la prise d'effet de sa mise en liberté ordonnée par le ministre en vertu de l'article 58.1 de la LIPR.

Le vide législatif concernant la durée maximale de la détention des mineurs étrangers devrait être comblé afin que la LIPR puisse se conformer à la norme internationale de protection des enfants qui exige de garantir un délai de détention aussi bref que possible. D'ailleurs, la Commission de l'immigration et du statut de réfugié (CISR) avait précisé qu'il existe une différence entre la détention d'un mineur et d'un non-mineur ${ }^{\mathrm{xxix}}$. C'est pourquoi nous ne voyons pas d'obstacles à ce que le législateur effectue un tel amendement à la LIPR.

Notons qu'en vertu de l'article 249 du Règlement sur l'immigration et la protection des réfugiés $(R I P R)^{x x x}$ les commissaires ou les agents de l'immigration doivent prendre en considération six critères réglementaires qui devraient être décidés dans l'intérêt supérieur de l'enfant ${ }^{x x i}$ au moment de la prise de décision de sa détention. Par ailleurs, lorsqu'il s'agit d'évaluer la détention d'un mineur pour le motif d'une identité non établie, les critères qui s'appliquent aux adultes ne doivent pas être les mêmes à l'égard d'un mineur ${ }^{\mathrm{xxxii}}$. C'est le cas par exemple du critère selon lequel l'étranger n'a pas coopéré. Les mineurs étrangers non 
accompagnés qui arrivent au Canada n'ont pas toujours avec eux les documents administratifs valides et ne coopèrent pas toujours adéquatement avec les autorités, non pas parce qu'ils ne veulent pas coopérer, mais simplement parce que leur degré de maturité ne leur permet pas de comprendre ce que les autorités leur demandent de fournir. L'absence de coopération peut aussi être due à la peur du mineur de parler, notamment s'il est victime d'exploitation ou d'enlèvement par des passeurs criminels.

\section{Conclusion}

Dans le présent article, nous avons mis en lumière les exigences de l'article 37 de la Convention relative aux droits de l'enfant, dont l'application s'étend à la détention des mineurs étrangers non accompagnés dans le contexte de l'immigration. En droit international des droits de l'enfant, la détention des mineurs étrangers non accompagnés n'est pas interdite en soi, mais cette mesure reste indésirable et doit être prise seulement si elle est nécessaire. Si finalement les autorités déclarent cette mesure nécessaire, elle doit alors être prise avec toutes les garanties relatives aux conditions de détention et aux droits procéduraux du mineur détenu.

Nous avons tenté d'examiner la conformité de la loi canadienne de l'immigration face à ces exigences; nous constatons deux lacunes majeures dans cette loi fédérale : le manque de disposition fixant une limite maximale pour la durée de détention des mineurs et l'inclusion des mineurs étrangers âgés de seize à dix-sept ans dans la définition de la nouvelle catégorie d'étrangers désignés. Cette inclusion a pour unique et regrettable conséquence de rendre obligatoire la détention de ces mineurs, ce qui contredit l'article 60 de la même loi. Enfin, il est souhaitable d'élaborer une base centrale de données statistiques relatives aux mineurs étrangers non accompagnés, comme le recommande le Comité des droits de l'enfant ${ }^{\mathrm{xx} x i i i}$.

Notes

${ }^{\mathrm{i}}$ Doctorante à la faculté de droit de l'université Laval

${ }^{i i}$ Loi du 15 décembre 1980 sur l'accès au territoire, le séjour, l'établissement et l'éloignement des étrangers, M.B. 20.12.2007.

${ }^{i i i}$ Cour eur. D.H., 12 octobre 2006, MubilanzilaMayeka et KanikiMitunga c. Belgique, (ciaprès: «Tabitha»). 


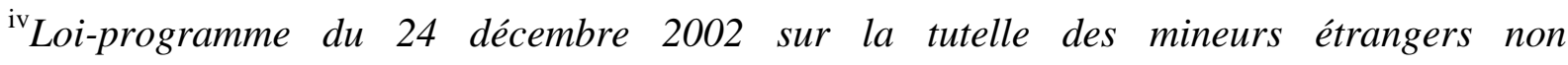
accompagnés, Moniteur Belge, 31.12.2002., Chapitre 6 - Tutelles des mineurs étrangers non accompagnés. La loi sur la tutelle est entrée en vigueur le $1^{\text {er }}$ mai 2004 conformément au prescrit de l'arrêté royal du 22 décembre 2003 portant exécution de la loi (M.B., 29.01.2004). Celui-ci fut modifié ensuite par l'arrêté royal du 7 décembre 2007 (M.B., 22.01.2008).

${ }^{\mathrm{v}}$ Convention relative aux droits de l'enfant du 20 novembre 1989, (1992) 1577 R.T.N.U 3 [1992] RT Can. n 3 (ci-après : CRDE).

${ }^{\mathrm{vi}}$ Nations unies, CRC/GC/2005/6, Observation générale $n^{\circ} 6$ (2005),traitement des enfants non accompagnés et des enfants séparés en dehors de leur pays d'origine, §7 (ci-après : O.G. $\left.n^{\circ} 6\right)$. Nous attirons l'attention du lecteur à ne pas confondre le mineur non accompagné avec le mineur séparé. Par mineur séparé on entend un enfant au sens de l'article $1^{\mathrm{er}}$ de la CRDE, lequel a été séparé de ses parents ou des personnes qui en avaient la charge à titre principal en vertu de la loi ou de la coutume, mais pas nécessairement séparé d'autres membres de sa famille. Le mineur séparé peut donc être accompagné par d'autres membres de sa famille, ce qui n'est pas le cas pour le mineur non accompagné. Cette distinction est adoptée aussi par le Haut Commissariat aux réfugiés et aux apatrides : Haut-commissariat des Nations Unies pour les réfugiés, Manuel de réinstallation - Département de la protection internationale, Genève novembre 2004, première éd. juillet 1997, p. IV/43.

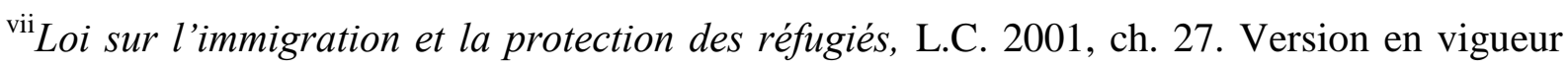
depuis le 6 févr. 2014 (ci-après : LIPR). Nous n'aborderons pas la détention en vertu du Code criminel canadien ou de la loi sur les jeunes contrevenants.

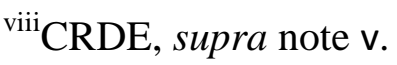

${ }^{\mathrm{ix}} \mathrm{CRC}$, O.G. $n^{\circ}$ 6, supra note vi, $\S 61$.

${ }^{\mathrm{x}} I d$.

${ }^{\mathrm{xi}}$ Le mineur détenu doit bénéficier des mêmes garanties procédurales minimales comme pour les adultes (article 9(2) et (4) Pacte international relatif aux droits civils et politiques et article 37(d) de la CRDE. Voir également, ONU, Ensemble de principes pour la protection de toutes les personnes soumises à une forme quelconque de détention ou d'emprisonnement, 1988; ONU, Règles minima pour le traitement des prisonniers, 1955. Il s'agit notamment du : $1^{\circ}$ droit de communiquer au mineur étranger non accompagné tout ordre de détention, incluant les raisons l'ayant motivé et les droits qui s'y rapportent; $2^{\circ}$ le droit d'être informé de son droit à un conseil juridique et à l'assistance juridique gratuite si elle peut être fournie; $3^{\circ}$ la possibilité que la décision de détention fasse l'objet de contrôles automatiques 
périodiques et réguliers par une instance judiciaire ou administrative indépendante de l'autorité chargée de la détention sur la nécessité de maintenir le mineur en détention. Le mineur ou son représentant devront pouvoir y assister. $4^{\circ}$ le droit de récuser, en personne ou par l'intermédiaire d'un représentant, la nécessité de la privation de liberté de l'enfant lors de l'audience de contrôle et de réfuter quelque verdict que ce soit. Enfin, $5^{\circ}$ le droit de contacter et d'être contacté par le bureau local du Haut Commissariat aux réfugiés et aux apatrides, par des institutions nationales pour les réfugiés ou par d'autres agences ainsi que par un avocat. ${ }^{x i i}$ Loi sur l'immigration et la protection des réfugiés, supra note vii.Voir, Wendy Ayotte, Separated children seeking asylum in Canada, juillet 2001, UNHCR-Canada, p. 19, en ligne :http://www.unhcr.ch: "There are no comprehensive statistics on detention of children, whether accompanied or not. CIC estimates show that during 1999-2000, 338 children were detained: 252 were held with a parent (usually the mother), 86 were separated children. Detention times varied: 252 were detained for one day or less; 24 were detained for less than 15 days; 42 were detained for over 30 days. CIC could not provide the age, nationality, sex, reason for the detention, or whether the children were refugee claimants. Approximately $50 \%$ were Chinese and Pakistani children. CIC suggested that most had been detained pending removal".

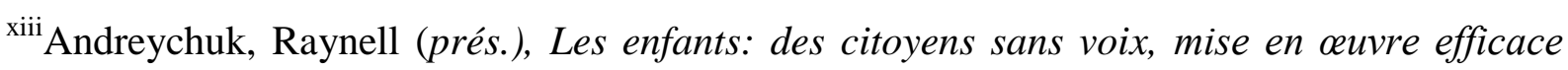
des obligations internationales du Canada relatives aux des enfants, Rapport final du Comité sénatorial permanent des Droits de la personne, en ligne: www.senate-senat.ca/rightsdroits.asp, p. 146-147.

${ }^{x i v}$ Caroline Montpetit, Des enfants dans les centres de détention, Le Devoir, 20 Janvier 2012, p. 1.

${ }^{\mathrm{xv}}$ Conseil canadien pour les réfugiés, La détention et l'intérêt supérieur de l'enfant, novembre 2009, p. 2-3, en ligne: www.ccrweb.ca.

${ }^{\mathrm{xvi}} \mathrm{CRC} / \mathrm{C} / 15 /$ Add.37, Observations finales du Comité des droits de l'enfant concernant le rapport initial du Canada, 20 juin 1995, p. 5. En ligne :http://tb.ohchr.org/default.aspx.

${ }^{x v i i}$ HCR, "Separated Children Seeking Asylum in Canada: A Discussion Paper Adapted From An Original Report, Researched and Written by Wendy Ayotte", july 2001, en ligne: http://ccrweb.ca, p. 22. Dans ce document il est précisé également qu'il n'existe pas de statistiques claires sur le nombre des mineurs étrangers non accompagnés détenus au Canada, mais le Service Citoyenneté et Immigration Canada avait estimé à 338 le nombre des mineurs détenus entre 1999-2000, Id., p. 19. 


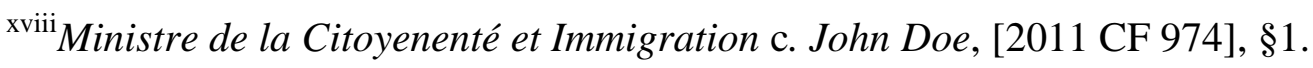

${ }^{\mathrm{xix}}$ LIPR, supra note, vii

${ }^{\mathrm{xx}}$ Avec ou sans mandat dépendamment du motif en question.

${ }^{\mathrm{xxi}}$ Sahin c. Canada (Ministre de la Citoyenneté et de l'Immigration), [1995] 1 C.F. 214 (1re inst.), appel rejeté dans Sahin, Bektas c. M.C.I. (C.A.F., A-575-94), Stone, MacGuigan, Robertson, 8 juin 1995.

${ }^{x x i i}$ Règlement sur l'immigration et la protection des réfugiés, DORS/2002-227(Règlement à jour au 14-05-2014). Il s'agit du motif de la détention, de la durée de la détention, de l'existence d'éléments permettant l'évaluation de la durée probable de la détention, des retards inexpliqués ou le manque inexpliqué de diligence de la part du ministère ou de l'intéressé et, de l'existence de solution de rechange à la détention.

${ }^{x x i i i}$ Voir également: Commission de l'immigration et du statut des réfugiés, Directives $n^{\circ} 2$ : Directives sur la détention, Directives données par le président en application de l'alinéa 159(1) (h) de la Loi sur l'immigration et la protection des réfugiés, en vigueur depuis le 29 octobre 2010 (mises à jour le 5 juin 2013), en ligne : http://www.irb-cisr.gc.ca. ${ }^{\mathrm{xxiv}} I d$., p. 12.

${ }^{\mathrm{xx}}$ Projet de Loi C-4 intitulé Loi modifiant la loi sur l'immigration et la protection des réfugiés, la loi sur les mesures de réforme équitables concernant les réfugiés et la loi sur la sûreté $d u$ transport maritime [dépôt et première lecture à la Chambre des Communes, le 16 juin 2012, $41^{\mathrm{e}}$ législature, $1^{\mathrm{re}}$ session), en ligne: www.parl.gc.ca.

${ }^{x x v i}$ Le Haut commissariat aux réfugiés et aux apatrides (HCR) considère indésirable la détention des enfants demandeurs d'asile qui sont en séjour irrégulier du fait qu'ils sont groupe vulnérable. HCR, Principes directeurs du HCR sur les critères et les normes applicables quant à la détention des demandeurs d'asile, Genève, 1999, p. 2, en ligne: http://www.unhcr.fr.

${ }^{x x v i i}$ Article 11 (1.1) LIPR.

xxviii Julie Béchard, Projet de Loi C-4 : Loi modifiant la loi sur l'immigration et la protection des réfugiés, la loi sur les mesures de réforme équitables concernant les réfugiés et la loi sur la sûreté du transport maritime, Publication $\mathrm{n}^{\circ}$ 41-1-C4-F, Bibliothèque du Parlement, 30 août 2011, p. 2. L'auteur examine les obligations internationales du Canada, mais elle ne mentionne pas la Convention relative aux droits de l'enfant parmi les instruments internationaux qu'elle nomme, voyez à la p. 2 et s. 
${ }^{\text {xxix }}$ Canada (Citoyenneté et de l'Immigration) c. Chi, 2001 CanLII 26665 (CISR). Il s'agissait du contrôle de la détention d'un adulte par la section d'arbitrage de la CISR selon laquelle : «En dernier lieu, le conseil a déposé, en tant que pièce D-1H, l'affaire Gao c. Canada. Je crois que le conseil n'a pas accordé beaucoup d'importance à cette décision et on le comprend aisément. Premièrement, cette affaire traite de mineurs et leur situation est sans aucun doute différente de celle des non-mineurs. Je remarque également que cette décision a été rendue par la Cour supérieure de l'Ontario qui, à en juger par le libellé et la teneur de la décision, ne traite pas nécessairement souvent de questions relatives à l'immigration. Je m'attendrais à ce que l'on accorde plus d'importance à une décision de la Cour fédérale comme Sahin, qui a certainement tenu compte, de façon soignée et détaillée, des conditions de détention à long terme de personnes en vertu de la Loi sur l'immigration».

${ }^{\mathrm{xx}}$ Règlement sur l'immigration et la protection des réfugiés (RIPR), supra note xxii.

xxxi Il s'agit des critères suivant : $1^{\circ}$ la possibilité d'arrangement avec les organismes ou les services d'aide à l'enfance en vue de protéger l'enfant au lieu de le détenir. $2^{\circ}$ La durée de la détention prévue. $3^{\circ}$ Le risque que le mineur demeure sous l'emprise des trafiquants qui l'ont amené au Canada. Ce critère peut être parfois compris par les autorités dans un sens qui favorise la détention des enfants afin de les «protéger» des trafiquants. Néanmoins, il doit y avoir des lieux d'hébergement alternatifs aux centres fermés. $4^{\circ}$ Le genre d'établissement de détention et les conditions de détention. $5^{\circ}$ La disponibilité des locaux permettant la séparation des mineurs des adultes détenus et, $6^{\circ}$ la disponibilité des services sociaux dans l'établissement de détention tels que des services d'éducation, d'orientation ou de loisirs.

${ }^{\mathrm{xxxii}} R I P R$, supra note xxii, paragraphe 247(2).

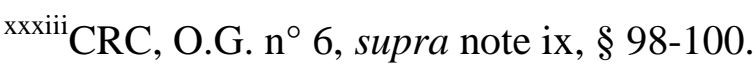

